\title{
0055 SAFETY STRATEGIES AND THE BALANCED SCORE CARD TECHNIQUE
}

P G Furst* Correspondence: Liberty Mutual Insurance Company, 12657 Alcosta Bulevard San Ramon, CA 94507, USA

\subsection{6/ip.2010.029215.55}

What you measure is what you get. For better or worse, measurement drives organisational behaviour to a large degree. For the most part the predominant measurement system utilised by management, in business, to assess success has been financial. In the mid-1980s structural changes in the economy and other business practices, started management looking for other means of information gathering and assessment to more successfully run their organisations.

Meanwhile other disciplines devised metrics with which to measure and affect business processes; the quality movement, reengineering, process management, to name a few. Also in the last 10-15 years change management and strategy implementation rose in prominence. The Balanced Scorecard provides a highly effective system with which to meet these challenges. This technique aligns organisational strategy and provides an in process, upstream measurement and improvement mechanism. 


\section{IP Safety 2010 abstracts}

Production quality and safety can greatly benefit from predictive metrics, especially safety whose metrics are reactive and out of alignment with business goals. In this session we will identify some of these roadblocks and explore methods with which safety performance can be effectively aligned with business strategy, made to drive desired behaviours, structurally remove organisational drives of loss and reduce the cost of risk. 